\title{
Variabilidade espacial da textura e da densidade de partículas em um solo aluvial no Município de Lavras, MG
}

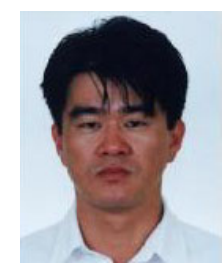

Edson S. Eguchi ${ }^{1}$, Elio L. da Silva ${ }^{2} \&$ Marcelo S. de Oliveira ${ }^{3}$

\author{
1 UFLA. Departamento de Engenharia. CEP 37200-000, Lavras, MG. E-mail: eguchi@ufla.br (Foto) \\ 2 UFLA. Departamento de Engenharia. Fone (35) 3829-1158. E-mail: elemos@ufla.br \\ 3 UFLA. Departamento de Ciências Exatas. Fone (35) 3829-1173. E-mail: marcelso@ufla.br
}

Protocolo $138-27 / 9 / 2001$

\begin{abstract}
Resumo: Para se de identificar a variabilidade espacial da textura e da densidade de partículas em um solo hidromórfico, realizou-se uma amostragem sistemática ao longo de uma transeção de 100 $\mathrm{m}$ de comprimento, paralela a um curso d'água, com espaçamentos entre pontos variando de 0,25 a $20 \mathrm{~m}$, totalizando 51 pontos amostrais. Utilizaram-se os métodos da estatística clássica e da geoestatística na identificação do tamanho e da estrutura da variabilidade. A percentagem de areia apresentou coeficiente de variação de $48,95 \%$, caracterizando alta variabilidade. Semivariogramas experimentais foram ajustados aos modelos esférico, Gauss e exponencial, com alcances de $15,0,49,5$ e $14,5 \mathrm{~m}$ para areia, argila e silte, respectivamente. Não foi possível um ajuste de semivariogramas para densidade de partículas. De modo geral, os resultados mostram que em solo aluvial os atributos texturais são espacialmente dependentes, mesmo, em transeção paralela ao curso d'água, considerada sujeita a condições semelhantes de sedimentação de partículas.
\end{abstract}

Palavras-chave: semivariograma, geoestatística, atributos do solo

\section{Spatial variability of the texture and particle density in an alluvial soil in the region of Lavras, MG - Brazil}

\begin{abstract}
The present study was carried out in order to identify the spatial variability of the texture and particle density in a hydromorphic soil. A systematic sampling in a $100 \mathrm{~m}$ transect was done, parallel to a small river, with spacing between sampling points varying from 0.25 to $20 \mathrm{~m}$, totaling 51 sample points. Classical statistical and geostatistical methods were used to identify size and structure of the variability. The sand fraction presented $48.95 \%$ as coefficient of variation, which characterizes high variability. Experimental semivariograms were fitted to spherical, Gauss and exponential models with ranges of 15.0, 49.5 and $14.5 \mathrm{~m}$ respectively for sand, clay and silt. It was not possible to adjust experimental semivariogram for particle density. The results, show that textural fractions are spatially dependent even in a transect parallel to a small river, subject to similar sedimentation.
\end{abstract}

Key words: semivariogram, geostatistics, soil formation, soil properties

\section{INTRODUÇÃO}

As várzeas, com 30 milhões de hectares irrigáveis, dos quais 10 milhões estão próximos aos grandes centros, constituem potencial para exploração agrícola. Devido a sua origem diversa quanto a granulometria, composição mineralógica e conteúdo de matéria orgânica, esses solos apresentam, normalmente, grande variação, com diferentes limitações e aptidão de uso. Este fato, aliado à falta de informações sobre suas propriedades e características, tem comprometido o sucesso dessas áreas, para a produção de alimentos (Machado, 1994).

O processo de formação das planícies de aluvionamento é bem conhecido. Segundo Telles (1988) quando ocorrem grandes chuvas, as águas em elevação estão sempre carregadas de elementos em suspensão e quando extravasam, abandonando a calha, perdem a capacidade de transporte e passam a depositálos. Esta sedimentação se faz, porém, de maneira bem regular, seguindo as leis de física de deposição em dois sentidos: na vertical e na horizontal, formando os chamados "leques aluviais".

Conforme Carvalho (1994) as partículas em suspensão estão sujeitas à ação de seu peso e, predominantemente, à ação da velocidade da corrente na direção horizontal, enquanto as partículas finas, como o silte e a argila, têm distribuição aproximadamente uniforme na vertical e as arenosas apresentam maior concentração próximo ao seu leito, formando a planície aluvionar.

De acordo com Telles (1988) as planícies aluvionares deveriam ter, numa seqüência horizontal e a partir das margens 
dos rios, solos arenosos, solos limosos e solos argilosos e, em profundidade e numa seqüencia vertical, os solos arenosos passam a ter camadas mais grosseiras e até cascalhentas ou pedregosas; os limosos passam a fino arenosos e arenosos, mas não arenosos grosseiros.

Os solos aluviais, afirmam Resende et al. (1988), são provenientes de depósitos aluviais e possuem horizonte escurecido sobre camadas estratificadas, muito variáveis a pequenas distâncias, tanto na horizontal como na vertical.

Segundo Libardi et al. (1986) os princípios básicos de experimentação e a variabilidade do solo ocorrem de forma aleatória; no entanto, vários estudos têm demonstrado que a variabilidade das propriedades físicas do solo apresenta dependência espacial. Para a caracterização de solos, a textura e a densidade de partículas são consideradas elementos de grande importância na tomada de decisão, na identificação e na classificação do solo, pois se constituem numa das características físicas mais estáveis.

O presente trabalho teve como objetivo a aplicação da estatística clássica e da geoestatística no estudo da variabilidade espacial da textura e da densidade de partículas de um solo aluvial, mostrando o seu comportamento ao longo de uma transeção paralela ao curso d'água.

\section{MATERIAL E MÉTODOS}

A área estudada localiza-se na margem direita do Ribeirão Santa Cruz, no município de Lavras, $\mathrm{MG}$, a $21^{\circ} 15^{\prime}$ ' de latitude sul e a $45^{\circ}$ de longitude oeste, com tipo climático, segundo a classificação de Koppen, Cwb, sendo utilizada atualmente como pastagem.

Segundo estudos realizados por Machado (1994) numa hidrosseqüência do espigão para o rio, a área compreende as classes de Latossolo Vermelho-Amarelo (LV) e os solos de várzea: Glei Pouco Húmico (HGP), Orgânico (HO) e o Aluvial.

A Figura 1 ilustra a amostragem de forma sistemática com espaçamento regular, variando de 0,25 a $20,00 \mathrm{~m}$, constituindo

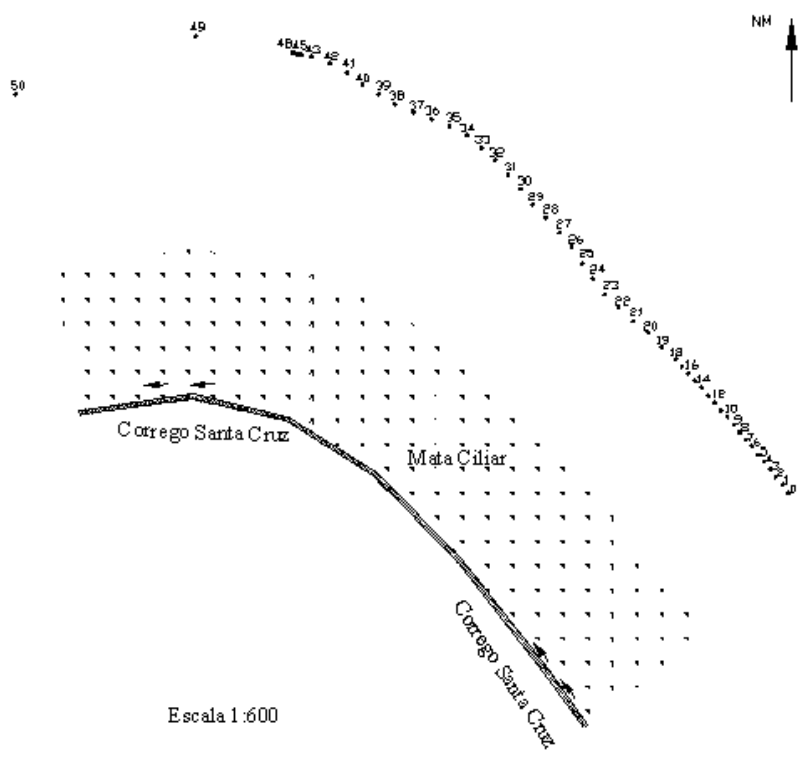

Figura 1. Esquema de amostragem na transeção uma transeção de $100 \mathrm{~m}$ de comprimento e um total de 51 pontos amostrais, com a transeção traçada em solo aluvial paralelamente ao curso d'água, a uma distância de $35 \mathrm{~m}$ de sua margem. Em cada ponto amostral procedeu-se à coleta de amostras deformadas, com trado de caneca na profundidade de $0,15 \mathrm{~m}$.

No Laboratório de Relação-Água-Solo-Planta, da Universidade Federal de Lavras, UFLA, determinou-se a densidade de partículas (Dp) através do método do picnômetro, descrita por Blake (1965) e a análise textural do solo através do método da pipeta descrita por Day (1965).

Todos os dados das variáveis em estudo foram armazenados em planilhas eletrônicas com o seu respectivo posicionamento geográfico. Usaram-se os recursos da estatística descritiva das inferências clássicas, através do software Sisvar e da geoestatística, por meio do software Variowin (Panatier, 1996) para o estudo da dependência espacial.

Seguindo-se critérios descritos por Libardi et al. (1996) a análise da estatística clássica dos dados foram realizadas, inicialmente, com as medidas de posição como a média, mediana, moda e média geométrica e, em seguida, com as medidas de dispersão, onde foram calculados os momentos de ordem até quatro, com a assimetria e curtose, que permitiu caracterizar a posição central e a dispersão dos dados. Foram determinadas, ainda, a distribuição gráfica da freqüência, seguindo critério de Scott (1979) e a normalidade das variáveis, verificadas pelo teste de Shapiro \& Wilk (1965).

A partir da hipótese de que cada valor medido é a realização de um processo estocástico no mínimo intrinsecamente estacionário, a geoestatística foi utilizada com o objetivo de se definir a estrutura de variabilidade espacial. Realizaram-se os cálculos das semivariâncias e os semivariogramas experimentais, cuja representação dos modelos foi mostrada por meio de gráficos da semivariância $\gamma(\mathrm{h})$ como função da distância (h). $\mathrm{O}$ estimador do semivariograma utilizado foi o "clássico" ou do método dos momentos, apresentado pela Eq. 1:

$$
\gamma(\mathrm{h})=\frac{1}{2 \mathrm{~N}(\mathrm{~h})} \sum_{\mathrm{i}=1}^{\mathrm{N}(\mathrm{h})}\left[\mathrm{Z}\left(\mathrm{x}_{\mathrm{i}}\right)-\mathrm{Z}\left(\mathrm{x}_{\mathrm{i}}+\mathrm{h}\right)\right]^{2}
$$

em que $\gamma$ é a semivariância experimental, obtida pelos valores amostrados $\left[\mathrm{Z}\left(\mathrm{x}_{\mathrm{i}}\right), \mathrm{Z}\left(\mathrm{x}_{\mathrm{i}}+\mathrm{h}\right)\right]$; $\mathrm{h}$ é a distância entre pontos amostrais e $\mathrm{N}(\mathrm{h})$ é o número total de pares de pontos possíveis, dentro da área de amostragem, com a distância h (Vieira, 2000).

\section{RESULTADOS E DISCUSSÃO}

Na classificação da USDA, pelos valores texturais médios encontrados, o solo se classificaria como franco. Pela análise da Figura 2, percebe-se acentuada variação textural do solo, ao longo da transeção. Constatam-se teores de areia superiores aos teores de silte e argila até a distância de $40 \mathrm{~m}$, ponto a partir do qual e até o final da transeção, os teores de silte superam a areia e a argila. Dos 40 aos $44 \mathrm{~m}$ da transeção, os teores de areia e de argila praticamente se igualam sendo, porém, inferiores aos teores de silte e, a partir dos $44 \mathrm{~m}$ verifica-se, ainda, que os teores de argila superam os teores de areia. Por ser a distância do curso d'água praticamente a mesma para todos os pontos 
amostrais que compõem a transeção, os coeficientes de variação dos atributos texturais deveriam ser baixos. A variação constatada pode ser devida a bloqueios naturais ora existentes nas diferentes épocas de inundação dissipando, em alguns trechos, a energia de arraste das partículas em suspensão que, com maior massa, tendem a se precipitar em um tempo menor e se distribuir geralmente próximo à calha.

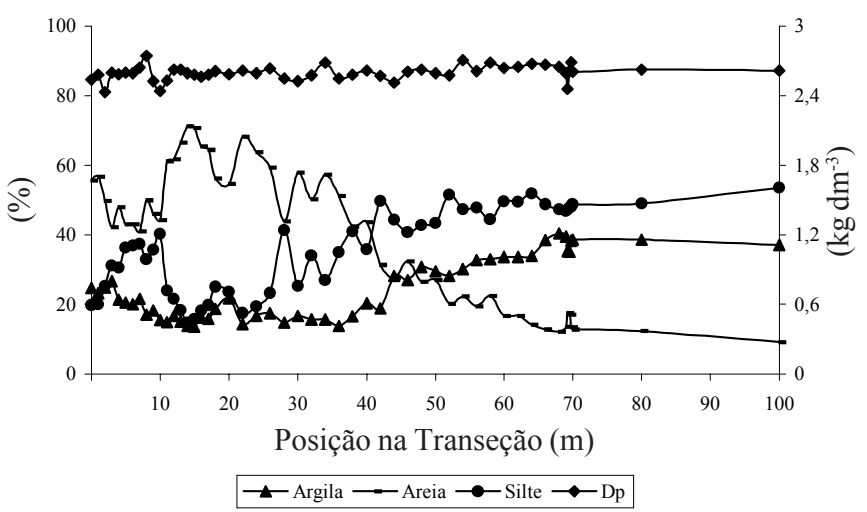

Figura 2. Valores referenciados da textura do soloe da densidade de partículas, medidos na transeção

Observa-se, na Tabela 1, que os valores das medidas de tendência central (média, mediana e moda) são semelhantes apenas para densidade de partículas, o que prova que a distribuição é simétrica para esse atributo do solo, confirmando Costa Neto (1990) e Libardi et al. (1996). As medidas de dispersão, próximas de 0 e 3 para assimetria e curtose, respectivamente, mostram distribuição normal, conforme Farias (1999). Pelos limites de coeficiente de variação propostos por Warrick \& Nielsen (1980) para a classificação de variáveis do solo $(\mathrm{CV}<12 \%),(12 \%<\mathrm{CV}>60 \%)$ e $(\mathrm{CV}>60 \%)$ para baixa, média e alta variabilidade, respectivamente, diz-se que os atributos texturais tiveram variabilidade média, e a densidade de partículas, uma variabilidade baixa.

Tabela 1. Estatística descritiva dos dados da areia, argila e silte, expressos em \% e densidade de partículas (Dp) $\mathrm{em} \mathrm{kg} \mathrm{dm}^{-3}$, ao longo da transeção

\begin{tabular}{lrrrc}
\hline \multirow{2}{*}{ Parâmetros Estatísticos } & \multicolumn{4}{c}{ Atributos } \\
\cline { 2 - 5 } & Areia & Argila & Silte & \multicolumn{1}{c}{ Dp } \\
\hline Média & 39,73 & 24,39 & 35,86 & 2,59 \\
Mediana & 44,66 & 22,81 & 38,08 & 2,60 \\
Moda & 50,97 & 16,39 & 47,11 & 2,60 \\
Média geométrica & 34,05 & 22,92 & 33,62 & 1,01 \\
Variância & 378,40 & 76,14 & 143,34 & 0,003 \\
Desvio-Padrão & 19,45 & 8,72 & 11,97 & 0,06 \\
Coeficiente de Variação & 48,95 & 35,76 & 33,38 & 2,38 \\
Assimetria & $-0,08$ & 0,42 & $-0,26$ & $-0,45$ \\
Curtose & 1,62 & 1,72 & 1,64 & 3,91 \\
Mínimo & 9,27 & 13,54 & 14,84 & 2,42 \\
Máximo & 71,20 & 40,38 & 53,57 & 2,74 \\
Amplitude Total & 61,93 & 26,83 & 38,72 & 0,31 \\
\hline
\end{tabular}

A área em que se desenvolveu o experimento vem sendo utilizada como pastagem natural, composta de vegetação variada, que pode ter influenciado na velocidade de arraste da

água e na sedimentação textural variada e no seu coeficiente de variação. Tal fato contraria, em parte, as afirmações de Guimarães (2000) de que as frações granulométricas são pouco dependentes do uso e manejo a que o solo é submetido, e que este atributo é fixo e a variação é proveniente da própria formação natural do solo.

Na Figura 3 são apresentados os histogramas, associados ao teste de normalidade (Shapiro \& Wilk, 1965) da argila, areia, silte e densidade de partículas. Pode-se visualizar a distribuição de freqüência dos atributos e a normalidade verificada apenas para densidade de partículas. Os resultados diferem daqueles obtidos por Nielsen et al. (1973) que observaram uma distribuição normal para argila e para areia e uma distribuição muito irregular e diferente da normal, para silte.

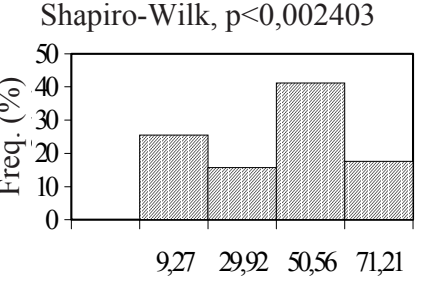

Areia (\%)

Shapiro-Wilk, $\mathrm{p}<0,001363$

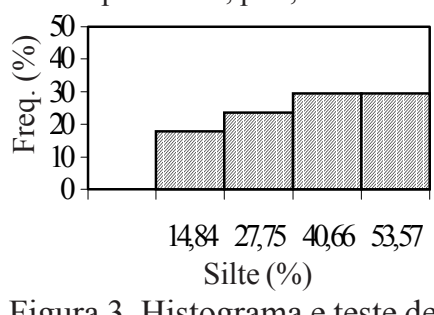

Figura 3. Histograma e teste de normalidade para argila, areia, silte e densidade de partículas (Dp) ao longo da transeção

Ante tal variação textural e visando à eficiência dos projetos de irrigação, do manejo e conservação ambiental do solo e água, que dependem intimamente desses atributos, seria errôneo considerar-se apenas os valores médios, visto que a sua distribuição não é normal.

$\mathrm{Na}$ Tabela 2, semivariogramas experimentais foram ajustados aos modelos esféricos, Gauss e exponencial, com alcances ( $a$ ) de 15,0, 49,5 e 14,5 m para areia, argila e silte, respectivamente. Para densidade de partículas não foi possível um ajuste. Nota-se grande diversidade nos modelos de semivariogramas ajustados para as variáveis relacionadas às amostras texturais, indicando que possíveis contribuições presentes na formação do solo, tenham influenciado na estrutura do semivariograma. Segundo

Tabela 2. Modelos de semivariogramas ajustados aos dados experimentais de areia, argila e silte em \% e de densidade média de partículas (Dp) $\mathrm{em} \mathrm{kg} \mathrm{dm}^{-3}$

\begin{tabular}{llcccc}
\hline \multirow{2}{*}{ Atributos } & \multicolumn{5}{c}{ Geoestatística } \\
\cline { 2 - 6 } & Modelo $^{*}$ & $\mathrm{C}_{0}$ & $\mathrm{C}_{0}+\mathrm{C}_{1}$ & $\mathrm{a}^{*}$ & $\mathrm{C}_{0} /\left(\mathrm{C}_{0}+\mathrm{C}_{1}\right)^{*}$ \\
\hline Areia & ESF & 3,47 & 132,72 & 15,00 & 2,61 \\
Argila & GAU & 2,97 & 128,33 & 49,50 & 2,31 \\
Silte & EXP & 0,10 & 74,76 & 14,50 & 0,14 \\
Dp & S/A & - & - & - & - \\
\hline
\end{tabular}

ESF - Modelo esférico; GAU - Modelo de Gauss; EXP - Modelo exponencial; S/A - Sem ajuste $\mathrm{C}_{0}$ - Efeito pepita; $\mathrm{C}_{0}+\mathrm{C}_{1}$ - Patamar; $a$ - Alcance $(\mathrm{m}) ; \mathrm{C}_{0} /\left(\mathrm{C}_{0}+\mathrm{C}_{1}\right)$ - Efeito pepita relativo (\%) 
a classificação de Cambardella et al. (1994) esses semivariogramas mostram um nível forte de dependência espacial, com relação ao efeito pepita $\left(\mathrm{C}_{0}\right) /$ patamar $\left(\mathrm{C}_{0}+\mathrm{C}_{1}\right)$ menor ou igual a $25 \%$.

$\mathrm{Na}$ Figura 4B, a maior dependência espacial encontrada para a argila neste solo, está de acordo com as afirmações de Isaaks \& Srivastava (1989) os quais chamam a atenção para o fato de que o comportamento parabólico do modelo de Gauss próximo à origem, é reflexo de boa continuidade, ou seja, a dependência entre amostras é alta, resultando maior alcance encontrado dentre os atributos estudados.

$\mathrm{Na}$ Figura 4D, apesar de não se encontrar um ajuste de um modelo teórico para a densidade de partículas, verificou-se que existe forte tendência para se atingir o efeito pepita puro ou a
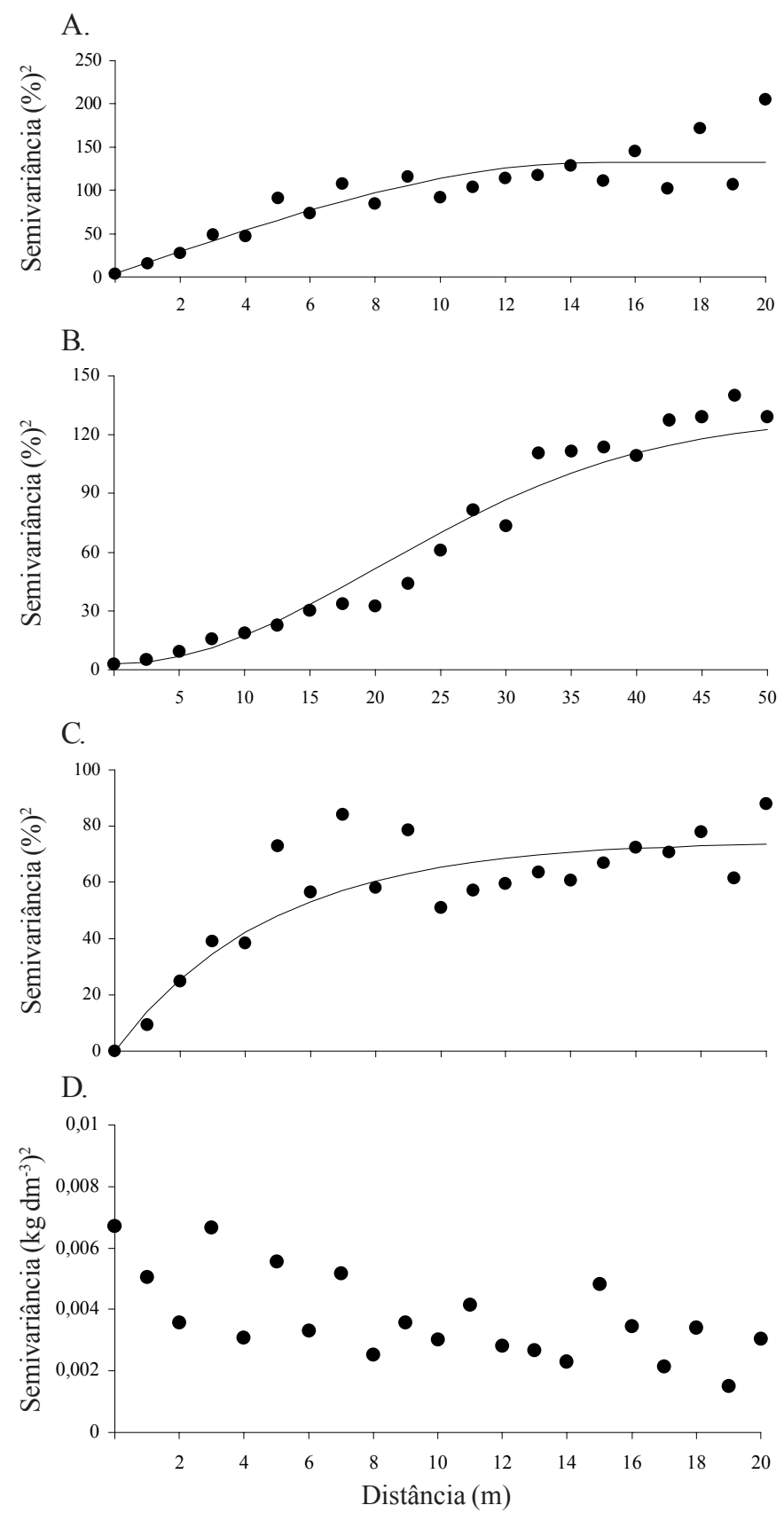

Figura 4. Semivariograma experimental para areia (A), argila (B), silte (C) e densidade de partículas (D) ausência total de dependência espacial. Segundo Vieira (2000) significa que o alcance (a), para os dados em questão, é menor que o menor espaçamento entre amostras. Conforme Silva (1988) para esses dados tem-se uma distribuição completamente aleatória e a única estatística aplicável é a estatística clássica.

\section{CONCLUSÕES}

1. A grande diversidade de estrutura de variabilidade mostra que o paralelismo a um curso d'água não define, por si só, a textura do solo; existe variação textural do solo ao longo de uma transeção.

2. Não é correto considerar-se os valores médios obtidos de amostras para classificação textural de um solo hidromórfico.

3. Os alcances ajustados aos semivariogramas experimentais correspondem às distâncias mínimas sugeridas entre amostras independentes para os separados texturais do solo avaliado.

4. Existe uma contrariedade na afirmação de que as frações granulométricas são pouco dependentes do uso e manejo do solo e que este atributo é fixo e a variação é proveniente da própria formação natural do solo.

5. A ausência total de dependência espacial para densidade de partículas determina que a única estatística aplicável para avaliação deste atributo é a estatística clássica.

\section{LITERATURA CITADA}

Blake, G.R. Bulk density. In: Black, C.A. (ed.) Methods of soil analysis; physical and mineralogical properties, including statistics of measurement and sampling. Madison: American Society of Agronomy, Pt.1, 1965. p.374-390.

Cambardella, C.A.; Moorman, T.B.; Novak, J.M.; Parkin, T.B.; Karlen, D.L.; Turco, R.F.; Konopka, A.E. Field-scale variability of soil properties in Central lowa soils. Soil Science Society of America Journal, Madison, v.58, n.5, p.1501-1511, 1994.

Carvalho, N.O. Hidrossedimentologia prática. Rio de Janeiro: CPRM, 1994.372p.

Costa Neto, P.L.O. Estatística. 10. ed. São Paulo: Edgard Blücher, 1990. 264p.

Day, P.R. Particle fractionation and particle size analysis. In: Black, C.A. (ed.) Methods of soil analysis: Physical and mineralogical properties, including statistics of measurement and sampling. Madison: American Society of Agronomy, Pt.1, 1965. p.545-567.

Farias, M.S.S. de. Diagnóstico da necessidade de drenagem no perímetro irrigado de São Gonçalo. Campina Grande: UFPB, 1999. 75p. Dissertação Mestrado

Guimarães, E.C. Variabilidade espacial de atributos de uma Latossolo Vermelho-escuro, textura argilosa, da região do cerrado, submetido ao plantio direto e ao plantio convencional. Campinas: UNICAMP, 2000, 85p. Tese Doutorado

Isaaks, E.H.; Srivastava, R.M. Applied geoestatistics. An introduction to. New York: Oxford University Press, 1989. 561p.

Libardi, P.L.; Manfron P.A.; Moraes, S.O.; Tuon, R.L. Variabilidade da umidade gravimétrica de um solo hidromórfico. Revista Brasileira de Ciência do Solo. Campinas, v.20, p.1-12, 1996. 
Libardi, P.L.; Prevedello, C.L.; Pauletto, E.A.; Moraes, S.O. Variabilidade espacial da umidade, textura e densidade de partículas ao longo de uma transeção. Revista Brasileira de Ciência do Solo, Campinas, SP, v.10, n.2, p.85-90, 1986.

Machado, R.V. Variabilidade espacial de atributos físicohídricos em uma hidrosseqüência de solos bem a muito mal drenados. Lavras: ESAL, 1994, 88p. Dissertação Mestrado

Nielsen, D.R.; Biggar, J.W.; Ehr, K.T. Spatial variability of field-measured soil water properties. Hilgardia, Berkeley, v.42, p.215-259, 1973.

Panatier, Y. Variowin: Software for spatial data analysis. 2. ed. New York: Springer-Verlag, 1996, 93p.

Resende, M.; Curi, N.; Santana, D. P. Pedologia e fertilidade do solo: Interações e aplicações. Brasília: Ministério da Educação; Lavras: ESAL; Piracicaba: POTAFOS, 1988, 81p.
Scott, D.W. On optimal and data based histograms. Biometrika, London, v.66, n.3, p.605-610, 1979.

Shapiro, S.S.; Wilk, M.B. An analysis of variance test for normality (complete sample). Biometrika, London, v.52, n.3/4, p.591-611, 1965.

Silva, A.P. Variabilidade espacial de atributos físicos do solo Piracicaba: ESALQ, 1988. 105p. Tese Doutorado

Telles, D. D. Drenagem. In: Módulo 2.3, Curso de Elaboração de Projetos de Irrigação. Brasília: ABEAS, 1988. 61p.

Vieira, S.R. Geoestatística em estudo de variabilidade espacial do solo. Tópicos em Ciência do Solo, Viçosa: Sociedade Brasileira de Ciência do solo. v.1, p.1-55, 2000.

Warrick, A.W.; Nielsen, D.R. Spatial variability of soil physical properties in the field. In: Hillel, D.(ed.). Applications of soil physics. New York: Academic Press, 1980. 